\title{
Distinct Effects of Brief and Prolonged Adaptation on Orientation Tuning in Primary Visual Cortex
}

\author{
Carlyn A. Patterson, ${ }^{1}$ Stephanie C. Wissig, ${ }^{1}$ and Adam Kohn ${ }^{1,2}$ \\ ${ }^{1}$ Dominick Purpura Department of Neuroscience and ${ }^{2}$ Department of Ophthalmology and Visual Sciences, Albert Einstein College of Medicine, Bronx, New \\ York 10461
}

Recent stimulus history-adaptation - alters neuronal responses and perception. Previous electrophysiological and perceptual studies suggest that prolonged adaptation strengthens and makes more persistent the effects seen after briefer exposures. However, no systematic comparison has been made between the effects of adaptation lasting hundreds of milliseconds, which might arise during a single fixation, and the more prolonged adaptation typically used in imaging and perceptual studies. Here we determine how $0.4,4$, and $40 \mathrm{~s}$ of adaptation alters orientation tuning in primary visual cortex of anesthetized macaque monkeys, and how quickly responses recover after adapter offset. We measured responses to small $\left(1.3^{\circ}\right)$ and large $\left(7.4^{\circ}\right)$ gratings because previous work has shown that adaptation effects can depend on stimulus size. Adaptation with small gratings reduced responsivity and caused tuning to shift away from the adapter. These effects strengthened with more prolonged adaptation. For responses to large gratings, brief and prolonged adaptation produced indistinguishable effects on responsivity but caused opposite shifts in tuning preference. Recovery from adaptation was notably slower after prolonged adaptation, even when this did not induce stronger effects. We show that our results can be explained by an adaptationinduced weakening of surround suppression, the dynamics of this suppression, and differential effects of brief and prolonged adaptation across response epochs. Our findings show that effects do not simply scale with adaptation duration and suggest that distinct strategies exist for adjusting to moment-to-moment fluctuations in input and to more persistent visual stimuli.

\section{Introduction}

The brain is altered by experience, allowing an organism to adjust to its environment. Experience-driven plasticity occurs over a range of timescales. Development unfolds over days to years and involves stable and enduring structural changes to brain circuits (Hensch, 2005). Perceptual learning in adulthood involves improvements typically seen over hours to weeks of training (Fahle and Poggio, 2002). More immediate experience-or adaptation-can also strongly alter neuronal responses.

The term adaptation is used to describe the experience of the preceding tens of milliseconds to several hours, and its effects have been documented in a large number of neural structures (Clifford et al., 2007; Kohn, 2007; Wark et al., 2007; Webster, 2011). Both perceptual and neurophysiological studies have provided evidence that similar effects are induced by brief and prolonged adaptation, with lengthier exposures simply strengthening and making more persistent effects seen after briefer

Received July 13, 2012; revised 0ct. 22, 2012; accepted 0ct. 31, 2012.

Author contributions: C.A.P. and A.K. designed research; C.A.P. and S.C.W. performed research; C.A.P. analyzed data; C.A.P., S.C.W., and A.K. wrote the paper.

This work was supported by National Institutes of Health Grants EY016774 and P3OHD071593 and the Research to Prevent Blindness. C.A.P. and S.C.W. were supported in part by National Institutes of Health Medical Scientist Training Program Training Grant T32-GM007288. We thank Amin Zandvakili, Xiaoxuan Jia, and Seiji Tanabe for assistance with data collection. We thank Steve Engel, Norma Graham, and Odelia Schwartz for helpful comments on a previous version of this manuscript.

Correspondence should be addressed to Adam Kohn or Carlyn Patterson, Dominick Purpura Department of Neuroscience, Albert Einstein College of Medicine, Kennedy 822, 1410 Pelham Parkway South, Bronx, NY 10461, E-mail: adam.kohn@einstein.yu.edu or carlyn.patterson@med.einstein.yu.edu.

DOI:10.1523/JNEUROSCI.3345-12.2013

Copyright $\odot 2013$ the authors $\quad 0270-6474 / 13 / 330532-12 \$ 15.00 / 0$ ones-a phenomenon we refer to as duration scaling (Bao and Engel, 2012). In the tilt aftereffect (TAE), for instance, an oriented stimulus viewed after an adapter appears repelled away. This occurs after adaptation as brief as $300 \mathrm{~ms}$ (Harris and Calvert, 1989), but the aftereffect is substantially stronger and longer lasting after viewing an adapter for $30 \mathrm{~min}$ (Magnussen and Johnsen, 1986). Similarly, neuronal contrast sensitivity in primary visual cortex (V1) is reduced after $50 \mathrm{~ms}$ adaptation (Bonds, 1991) but more strongly after 80 s adaptation (Ohzawa et al., 1985). Adaptation with a stimulus that falls on the flank of a tuning curve causes a repulsive shift in preference, after exposures as brief as $50 \mathrm{~ms}$ (Felsen et al., 2002) and as prolonged as 10 min (Dragoi et al., 2000, 2001).

However, systematic studies of the influence of adaptation duration, although rare, suggest a more complex picture than the duration scaling description. Perceptual aftereffects can be as strong after brief as prolonged adaptation (Harris and Calvert, 1989; Foley and Boynton, 1993), and some aftereffects may only be induced by brief adapters (Suzuki, 2001, 2005). Neurophysiological studies have revealed that some changes in tuning are only recruited after prolonged (many tens of seconds) adaptation (Dragoi et al., 2000; Sharpee et al., 2006). Furthermore, neuronal contrast sensitivity can be affected more strongly by brief dynamic adapters than more prolonged but constant ones (Crowder et al., 2008).

Determining how adaptation duration influences changes in neuronal response properties is central to understanding whether sensory systems use distinct strategies for adjusting sensitivity on different timescales. Here we test the adequacy of duration scal- 
ing as a description of adaptation-induced changes in orientation tuning in macaque $\mathrm{V} 1$. We measured the effects of $0.4,4$, and $40 \mathrm{~s}$ adaptation with small and large sinusoidal gratings. Our results show that brief and prolonged adaptation can lead to qualitatively different changes in tuning and thus that duration scaling is a poor description of adaptation effects. Rather, distinct strategies are used to adjust the cortical representation to moment-to-moment fluctuations in input and to more persistent visual stimuli.

\section{Materials and Methods}

We implanted 15 arrays, in nine male monkeys (Macaca fascicularis). Before surgery, monkeys were administered $0.05 \mathrm{mg} / \mathrm{kg}$ atropine and 1.5 $\mathrm{mg} / \mathrm{kg}$ diazepam. Anesthesia was induced with ketamine $(10 \mathrm{mg} / \mathrm{kg})$, and the animal was intubated and administered $1.0-2.5 \%$ isoflurane in a $98 \%$ $\mathrm{O}_{2} / 2 \% \mathrm{CO}_{2}$ mixture. An intravenous catheter was placed in the saphenous vein of each leg. The animal was then positioned in a stereotaxic device, and a craniotomy and durotomy were performed over V1. A $10 \times$ 10 microelectrode array (400 $\mu \mathrm{m}$ spacing, $1 \mathrm{~mm}$ length; Blackrock Microsystems) was implanted, and the brain was covered with agar to prevent desiccation. Anesthesia during recordings was maintained with a venous infusion of sufentanil citrate $\left(6-18 \mu \mathrm{g} \cdot \mathrm{kg}^{-1} \cdot \mathrm{h}^{-1}\right.$, adjusted as needed) in Normosol solution with dextrose. The monkey was paralyzed with vecuronium bromide $\left(0.15 \mathrm{mg} \cdot \mathrm{kg}^{-1} \cdot \mathrm{h}^{-1}\right)$ to minimize eye movements. Vital signs - electrocardiogram, blood oxygen saturation, blood pressure, EEG, end-tidal $\mathrm{CO}_{2}$ partial pressure, temperature, and airway pressure-were constantly monitored to ensure adequate anesthesia and animal well being. Topical atropine was used to dilate the pupils, and corneas were protected with gas-permeable contact lenses. A broad-spectrum antibiotic (Baytril, $2.5 \mathrm{mg} / \mathrm{kg}$ ) and an antiinflammatory steroid (dexamethasome, $1 \mathrm{mg} / \mathrm{kg}$ ) were administrated daily. Supplementary lenses were used to bring the retinal image into focus. All procedures were approved by the Institutional Animal Care and Use Committee of the Albert Einstein College of Medicine at Yeshiva University and were in compliance with the guidelines set forth in the National Institutes of Health Guide for the Care and Use of Laboratory Animals.

Stimuli. Stimuli were generated with custom software based on OpenGL (EXPO) and displayed on a calibrated cathode ray tube monitor ( $1024 \times 768$ pixels; $100 \mathrm{~Hz}$ frame rate; $\sim 40 \mathrm{~cd} / \mathrm{m}^{2}$ mean luminance). We measured the spatial receptive field (RF) of each unit by the sequential, pseudorandom presentation of small gratings $\left(0.5^{\circ}\right.$ diameter; four orientations; $250 \mathrm{~ms}$ presentation) at a range of locations that spanned a $3 \times 3^{\circ}$ area. We used these measurements to center our stimuli over the aggregate RF of the sampled units and, when considering responses to small gratings, to select units whose RF was at least $50 \%$ covered by the stimulus for further analysis.

We measured tuning with 16 equally spaced orientations of drifting sinusoidal gratings $\left(1 \mathrm{cycle} /{ }^{\circ}\right.$, drifting at $6.25 \mathrm{~Hz}, 22.5^{\circ}$ steps $)$, in control (preadaptation) conditions and after presenting one grating as an adapter for $0.4,4$, or $40 \mathrm{~s}$. Gratings were either 1.3 or $7.4^{\circ}$ in diameter, with the adapter and test gratings matched in size. To provide similar sample sizes, we ran more experiments with small gratings because fewer neurons were driven by these stimuli. Data were generally collected in a single block, with the different conditions (stimulus size and adaptation duration) presented in a pseudorandom sequence and an interval sufficient to allow full recovery between conditions.

For adaptation durations of 0.4 and $4 \mathrm{~s}$, the preadaptation and postadaptation conditions were interleaved (intertrial interval of 1.2 and $12 \mathrm{~s}$, respectively; Fig. 1A). Preadaptation responses were measured with stimuli preceded by a blank screen of mean luminance, presented for the same duration as the adapter. For $40 \mathrm{~s}$ adaptation, an adapt-test-top-up paradigm was used (Kohn and Movshon 2004; Fig. 1A), with $1 \mathrm{~s}$ test stimuli and $5 \mathrm{~s}$ top-up presentations of the adapter. Preadaptation test stimuli were separated by a $5 \mathrm{~s}$ interstimulus interval to maintain the same temporal structure as the postadaptation measurements. A portion of the $40 \mathrm{~s}$ adaptation data have been presented previously (Wissig and Kohn, 2012, their Fig. 7). We used a $1 \mathrm{~s}$ test stimulus after 4 and $40 \mathrm{~s}$ adaptation, and a $0.4 \mathrm{~s}$ test stimuli after $0.4 \mathrm{~s}$ adapters, to allow compar- isons to other stimulus conditions not discussed here. To ensure fair comparisons across conditions, all analysis was performed using a common $350 \mathrm{~ms}$ epoch, beginning $50 \mathrm{~ms}$ after stimulus onset to account for response latency.

We measured the time course of recovery after $0.4,4$, and $40 \mathrm{~s}$ adaptation, using identical large $\left(7.4^{\circ}\right)$ adapter and test gratings. For $0.4 \mathrm{~s}$ adaptation, the adapter was followed on each trial by a single $0.4 \mathrm{~s}$ test stimulus after a delay ranging from 0 to $0.8 \mathrm{~s}$. Trials were separated by an interval of $1.6 \mathrm{~s}$. For $4 \mathrm{~s}$ adaptation, the adapter was followed by a single $0.4 \mathrm{~s}$ test after a delay ranging from 0 to $12 \mathrm{~s}$; trials were separated by $24 \mathrm{~s}$. For $40 \mathrm{~s}$ adaptation, the adapter was followed on each trial by multiple $1 \mathrm{~s}$ test presentations at delays ranging from 0 to $120 \mathrm{~s}$; trials were separated by $130 \mathrm{~s}$.

Data analyses. Neural signals that exceeded a user-defined threshold were digitized at $30 \mathrm{kHz}$ and sorted offline into single-unit and multiunit activity (Plexon Offline Sorter). Responses were measured as the F1 component of the response or the mean firing rate, depending on which was larger. Evoked responses (spontaneous activity subtracted) were analyzed for all units whose peak measured response was greater than the mean $+1 \mathrm{SD}$ of the spontaneous firing rate.

Tuning curves were folded to span $0-180^{\circ}$ and fit with a von Mises, or circular Gaussian, function:

$$
r_{p}(\theta)=m+a e^{b\left(\cos \left(\theta-\theta_{\text {pret }}\right)-1\right)}
$$

where $r_{p}$ is the predicted response and $\theta$ is the direction; $m$ determines the baseline offset, $a$ the peak response amplitude, $b$ the tuning bandwidth, and $\theta_{\text {pref }}$ the preferred orientation. Tuning curves were fit by maximizing the log likelihood of obtaining the data given the model (i.e., von Mises function) predictions, under the assumption of Poisson spiking statistics (El-Shamayleh and Movshon, 2011). Fit quality was calculated as a "normalized log likelihood": the lower bound (value of 0 ) consisted of the likelihood of a model with predicted responses equal to the mean response averaged across all conditions, and the upper bound (value of 1) consisted of using the measured responses as the model (Stocker and Simoncelli, 2006; ElShamayleh and Movshon, 2011).

We discarded units with the following criteria: (1) units with a preadaptation and postadaptation fit quality $<0.5$ ( $29 \%$ of cases; average fit quality in the remaining units was 0.87); (2) units with a bandwidth $<22.5^{\circ}$ (defined as the full width of the tuning curve midway between the maximum and minimum evoked response; $10 \%$ of remaining cases), because we could not accurately measure tuning for units with a bandwidth smaller than our sampling of orientation; (3) units whose orientation shift was $>0.75$ of the bandwidth of the unit $(0.8 \%$ of remaining cases) because previous studies suggest that such shifts likely indicate the preadaptation and postadaptation data originated from different cells (Dragoi et al., 2000; Kohn and Movshon, 2004). For analysis of response dynamics, these same criteria were applied to tuning in each time epoch.

We quantified the recovery from adaptation by fitting the data with a single-term, rising exponential:

$$
r(t)=1-a e^{-t / \tau},
$$

where $r(t)$ is the response relative to the maximal response, measured at time $t$ after adapter offset, $\tau$ is the time constant of recovery, and $a$ is a scaling factor. Fits were obtained using a $\chi^{2}$ minimization method. Data from the initial response epoch and later epochs were fit separately, as described in Results. A single-term rising exponential provided a better description of recovery than a sum of two rising exponentials $\left(r(t)=1-a_{1} e^{-t / \tau_{1}}-a_{2} e^{-t / \tau_{2}}\right)$ or a power function $\left[r(t)=a t^{n}\right]$, when the number of free parameters in each descriptive function was taken into account ( $\chi^{2}$ value weighted by degrees of freedom in the model).

Error bars indicate $95 \%$ confidence intervals based on bootstrap analysis using the percentile method (i.e., the range of the 2.5th to 97.5th percentile values), unless otherwise indicated. We used $t$ tests to determine statistical significance, unless stated otherwise. Ratios were logtransformed before statistical evaluation.

\section{Results}

We performed extracellular recordings in the parafoveal representation of V1. Arrays were implanted $\sim 600 \mu \mathrm{m}$ beneath the 
A $0.4 \mathrm{~s}$ adaptation

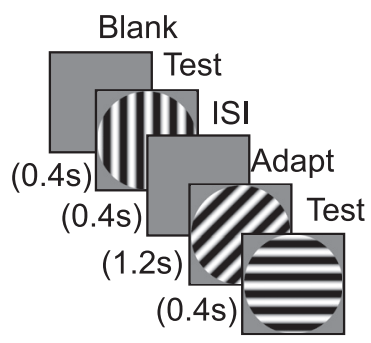

(0.4s)
4 s adaptation

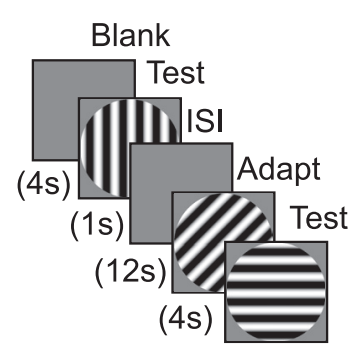

(1s)

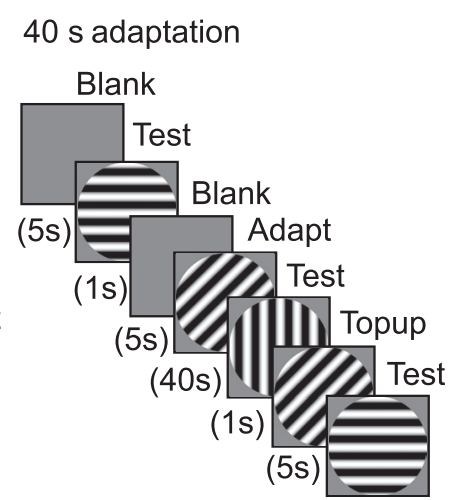

(1s)

\section{B}

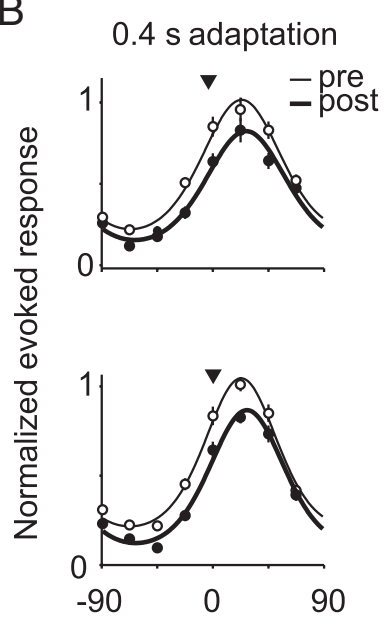

C
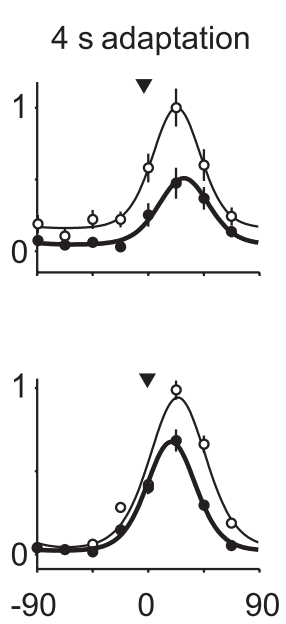

D
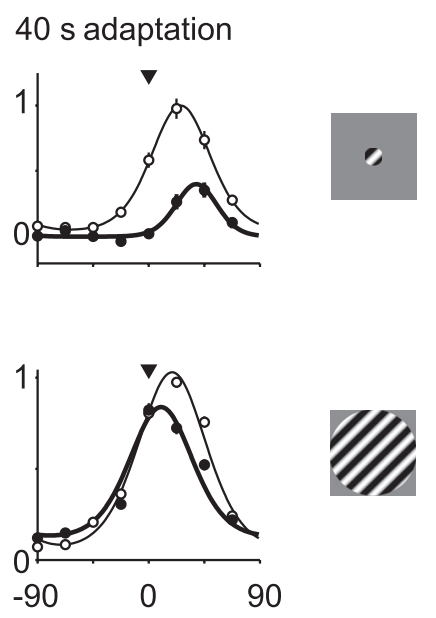

Orientation of test stimulus (deg)

Figure 1. A, Stimulus presentation protocols for $0.4,4$, and 40 s adaptation. $\boldsymbol{B}-\boldsymbol{D}$, Tuning curves of six example units for small (top) and large (bottom) gratings are shown before (thin lines; open symbols) and after (thick lines; filled symbols) $0.4 \mathrm{~s}$ adaptation (B), 4 s adaptation ( $\boldsymbol{C}$, and 40 s adaptation (D). Symbols indicate observed responses, and solid lines show fits to the data. Arrowhead indicates orientation of adapter. Error bars indicate SEM. Evoked responses of each unit were normalized by the maximum response across all conditions.

cortical surface (always $<1 \mathrm{~mm}$, the electrode length). We thus targeted primarily layers $2 / 3$ and $4 \mathrm{~B}$, in which neurons projecting to higher visual cortex are found (Felleman and Van Essen, 1991). We measured responses to gratings with a spatial frequency of 1 cycle $/^{\circ}$, drifting at $6.25 \mathrm{~Hz}$. These parameters were chosen to be appropriate for the preferences of parafoveal V1 neurons (Foster et al., 1985) and evoked measurable responses in most units.

Recordings consisted of both single-unit and multiunit activity, sorted offline. Effects were similar for these two types of recordings so the data were pooled (for detailed comparison of effects of prolonged adaptation in single-unit and multiunit recordings, see Wissig and Kohn, 2012). We refer to both types of response as arising from units.

We measured the effects of $0.4,4$, or $40 \mathrm{~s}$ adaptation on orientation tuning. For 0.4 and $4 \mathrm{~s}$ adaptation, we interleaved test stimuli preceded by the adapter (postadaptation) with those preceded by a screen of mean luminance (preadaptation), with a sufficient intertrial interval to ensure complete recovery. For $40 \mathrm{~s}$ adaptation, this protocol was impractical so we used an adapttest-top-up design (Fig. 1A; see Materials and Methods).

We measured responses to small $\left(1.3^{\circ}\right.$, a typical size of a parafoveal V1 RF; Cavanaugh et al., 2002) and large $\left(7.4^{\circ}\right)$ gratings, with the adapter and test stimuli always matched in size. We did so because the effects of prolonged adaptation on V1 orientation tuning have been shown to depend on stimulus size (Wissig and
Kohn, 2012). This was suggested to occur because adaptation causes a stimulus-specific reduction in responsivity to stimuli presented within the classical receptive field (CRF) and, for large stimuli only, a stimulus-specific reduction in the efficacy of surround suppression recruited from beyond the CRF (Angelucci and Bressloff, 2006). The weakening of surround suppression after adaptation is a form of disinhibition that can facilitate responses and cause tuning to shift toward the adapter.

The organization of this study is as follows. We first compare the effects of large and small adapters, presented for different durations, on V1 orientation tuning. This shows that effects depend on stimulus size, after prolonged but not brief adaptation. It also reveals that lengthier adaptation does not simply strengthen the effects seen after brief adaptation, contrary to the duration scaling description. In the following section, we analyze how adaptation-induced changes in tuning depend on the response epoch analyzed. This shows that brief adaptation affects primarily the initial response epoch, whereas more prolonged adaptation alters responses in later epochs. It is only in these later epochs that the influence of stimulus size is apparent, suggesting a delayed onset of surround suppression. We confirm this by measuring the dynamics of surround suppression, and we show that the strength of surround suppression predicts how prolonged adaptation alters tuning. Finally, we test whether prolonged adaptation results in more persistent adaptation effects. We find that it 
A

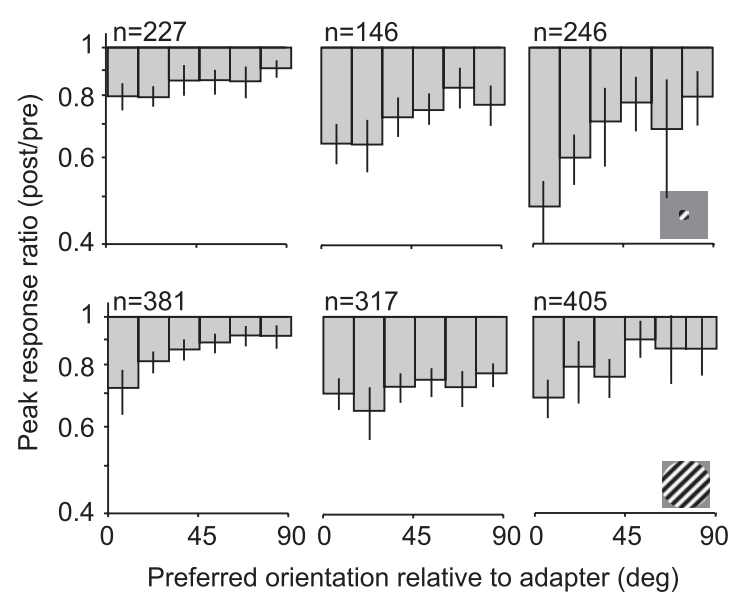

B

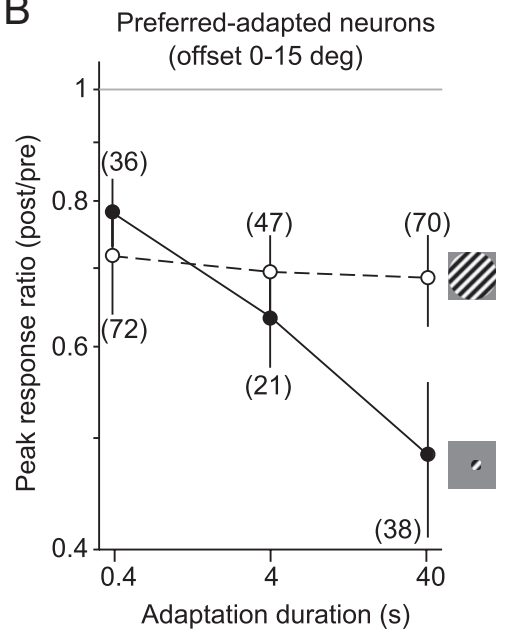

Figure 2. Changes in peak responsivity, as a function of stimulus size and adaptation duration. $A$, Peak response ratio (post/pre) as a function of neuronal orientation preference, for small (top) and large (bottom) gratings. Number of units, $n$, refers to the total, across all orientation preference offsets. $\boldsymbol{B}$, Peak response ratios for preferred-adapted neurons $\left(0-15^{\circ}\right.$ offset from adapter) as a function of adaptation duration, for small (solid) and large (dotted) gratings. Numbers in parentheses indicate number of units in each condition. Error bars indicate $95 \%$ confidence intervals.

does, even when those effects are not stronger than those observed after briefer adaptation. Furthermore, we find that different response epochs recover at distinct rates.

Effects as a function of adaptation duration and stimulus size Figure $1 B-D$ shows representative effects on orientation tuning curves for each stimulus size and adaptation duration (before adaptation in thin lines and open symbols; after adaptation in thick lines and filled symbols). For each of these six units, the adapter was presented on the flank of the tuning curve, offset $20-30^{\circ}$ from the preferred orientation. The $0.4 \mathrm{~s}$ adaptation caused repulsive shifts in orientation preference (away from the adapter) and a weak reduction in peak response, for both small (top) and large (bottom) gratings (Fig. $1 B$ ). After $4 \mathrm{~s}$ adaptation, tuning measured with small stimuli typically showed stronger repulsive shifts and a greater reduction in peak response (Fig. $1 C$, top). For tuning measured with large gratings, adaptation resulted in attractive shifts in preference with no evidence for a greater loss of responsivity (bottom). After $40 \mathrm{~s}$ adaptation, responses to small gratings were reduced further and tuning was strongly repelled from the adapter, whereas tuning for large gratings underwent an attractive shift and a weak reduction in responsivity (Fig. 1D).

To quantify adaptation-induced changes in tuning, we fit the preadaptation and postadaptation responses from each unit with a von Mises function (Fig. $1 B-D$, solid lines) and extracted the peak response, preferred orientation, and tuning bandwidth. The peak response ratio (after adaptation compared with before) for the full population of recorded cells is shown in Figure $2 \mathrm{~A}$. Geometric mean ratios are plotted as a function of the preferred orientation of the neurons relative to the adapter, because the effects of adaptation are known to depend on this offset (Müller et al., 1999; Dragoi et al., 2000, 2002; Kohn and Movshon, 2004; Wissig and Kohn, 2012). The response ratio depended on both stimulus size ( $F=11.3, p=0.0008$, two-way ANOVA) and adaptation duration $(F=30.1, p<0.0001)$, with a significant interaction between these $(F=11.3, p<0.0001)$.

For activity driven by small gratings, responsivity was more strongly reduced as adaptation duration increased, with the greatest effect seen for preferred-adapted neurons (offset by $<15^{\circ}$ from the adapter). For these neurons, whose data are replotted in
Figure $2 B$ to facilitate comparisons among conditions, the response ratio decreased from 0.78 after $0.4 \mathrm{~s}$ adaptation to 0.48 after $40 \mathrm{~s}$ adaptation $(p<0.0001)$. For responses to large gratings (Fig. $2 A$, bottom row; $B$, dotted line), the loss of responsivity was similar across adaptation durations: the average ratio was 0.72 after the briefest adaptation and 0.69 after the most prolonged $(p=0.5)$.

We next determined the influence of adaptation duration on shifts in preferred orientation. Shifts depended on stimulus size $(F=33.8, p<0.0001$, two-way ANOVA) but not duration $(F=$ $2.1, p=0.1$ ). This was because small and large stimuli yielded opposite trends, with a strong interaction between the influence of stimulus size and adaptation duration $(F=6.44, p=0.002)$. For activity driven by small gratings, shifts were repulsive for all adaptation durations (Fig. 3A, top row) and were most pronounced for units adapted on the tuning curve flank (offsets of $15-75^{\circ}$ from the adapter; replotted in Fig. $3 B$ ). For these units, there was a weak increase in strength with more prolonged adaptation, with the mean shift increasing from $3.3^{\circ}$ for the briefest to $5.1^{\circ}$ for the longest adaptation duration $(p=0.09)$. For large gratings (bottom row), brief adaptation caused significant repulsive shifts $\left(2.1^{\circ}, p<0.0001\right.$ for difference with 0$)$, whereas $40 \mathrm{~s}$ adaptation led to attractive shifts in preference $\left(-2.0^{\circ}, p=\right.$ 0.002 ). Thus, shifts in preference were qualitatively different after brief and prolonged adaptation, for activity driven by large gratings.

Note that the preadaptation responses were higher to small gratings than large ones (peak response of $23.2 \pm 1.4$ vs $16.3 \pm 1.2$ spikes/s, $p<0.0001)$. However, the strength of adaptation effects for a given stimulus size and adaptation duration were only weakly, and in many cases not significantly, related to response strength across units $(r<0.2$; data not shown; see also Wissig and Kohn, 2012). Thus, the different effects for large and small adapters are not attributable to differences in response rate between these conditions.

Finally, we measured the effect of adaptation on tuning bandwidth (Fig. 4). Across all conditions, bandwidths were slightly narrower (10-20\%) after adaptation than before. This effect was strongest for preferred-adapted neurons $(p<0.05$ for all conditions). However, the bandwidth ratio (after adaptation compared with before) for the full population did not depend on stimulus 
A

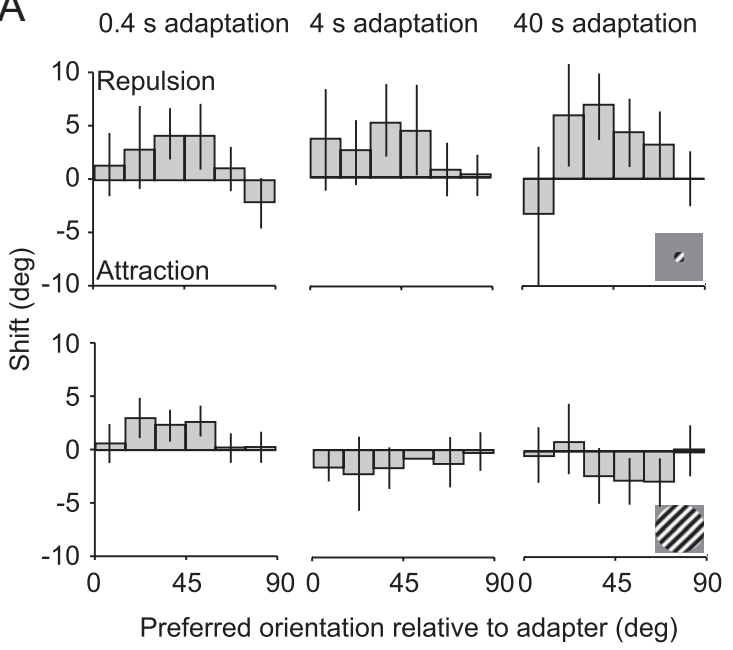

B

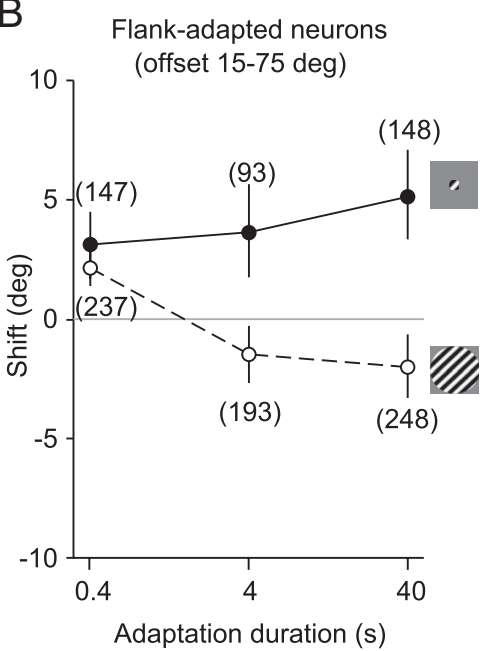

Figure 3. Shifts in preferred orientation, as a function of stimulus size and adaptation duration. $A$, Shifts in preferred orientation as a function of neuronal orientation preference, for small (top) and large (bottom) gratings. $\boldsymbol{B}$, Shifts for flank-adapted neurons ( $15-75^{\circ}$ offset from adapter) as a function of adaptation duration, for small (solid) and large (dotted) gratings. Error bars indicate $95 \%$ confidence intervals.

A 0.4 s adaptation 4 s adaptation 40 s adaptation

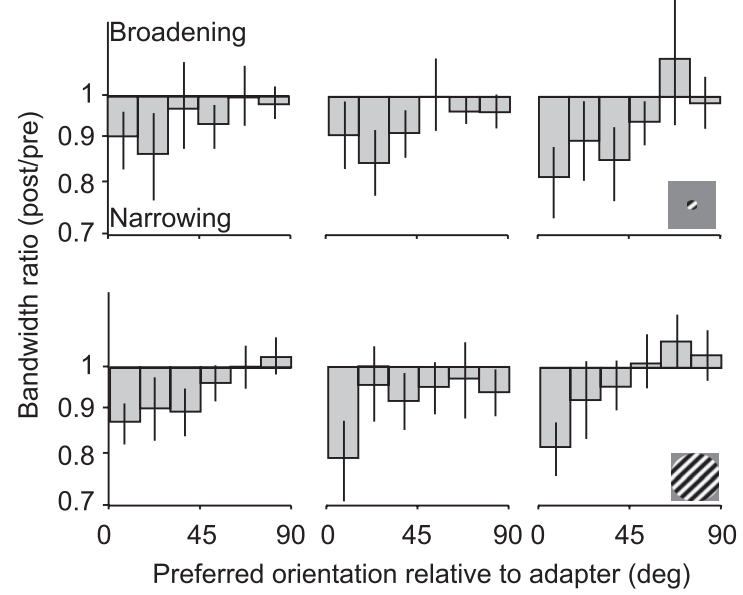

B

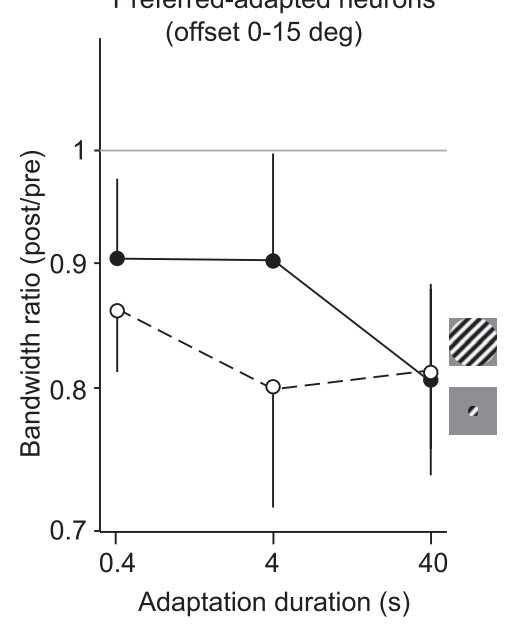

Figure 4. Changes in bandwidth, as a function of stimulus size and adaptation duration. $A$, Changes in orientation tuning bandwidth (post/pre) as a function of neuronal orientation preference, for small (top) and large (bottom) gratings. $\boldsymbol{B}$, Bandwidth ratios for preferred adapted units ( $0-15^{\circ}$ offset from the adapter) for small (solid) and large (dotted) gratings, as a function of adaptation duration. Error bars indicate $95 \%$ confidence intervals.

size $(F=0.4, p=0.5$, ANOVA $)$ or adaptation duration $(F=0.4$, $p=0.7$; interaction, $F=1.3, p=0.3)$. We did not analyze these effects further.

In summary, the effects of adaptation with small gratings were well characterized by duration scaling: repulsive shifts in preference and the reduction of responsivity became more pronounced with more prolonged adaptation. For adaptation with large gratings, this was not the case. Brief adaptation was as effective in reducing responsivity as adaptation that was 100 -fold longer, and shifts in preference were qualitatively different after brief (repulsive) and prolonged (attractive) adaptation.

\section{A comparison of tuning effects in different response epochs}

To gain insight into the effects observed after adaptation of different durations, we analyzed response dynamics. Brief adaptation has been shown to affect primarily the early epoch of the response or "onset transient" (Müller et al., 1999; Priebe and Lisberger, 2002; Priebe et al., 2002; Liu et al., 2009), but how different response epochs are affected by prolonged adaptation is unknown. Comparing adaptation effects in different epochs provided insight into why effects measured with small gratings scaled with adaptation duration but those measured with large gratings did not. It also revealed another violation of duration scaling.

We measured orientation tuning in early (50-100 ms after stimulus onset), mid (100-200 ms), and late (200-400 ms) response epochs, subdividing the response window (50-400 ms) on which the preceding analysis was based. To reduce the number of comparisons, we present changes in peak responsivity in preferred-adapted neurons after 0.4 and 40 s adaptation, because these caused the most distinct effects; effects of $4 \mathrm{~s}$ adaptation fell between those observed for these adaptation durations. For each epoch and adaptation duration, we analyzed effects only from units that were well-tuned in that epoch and for that condition, as defined in Materials and Methods.

After $0.4 \mathrm{~s}$ adaptation (Fig. $5 A$ ), peak responses were strongly reduced in the early epoch for activity driven by small (geometric 
A

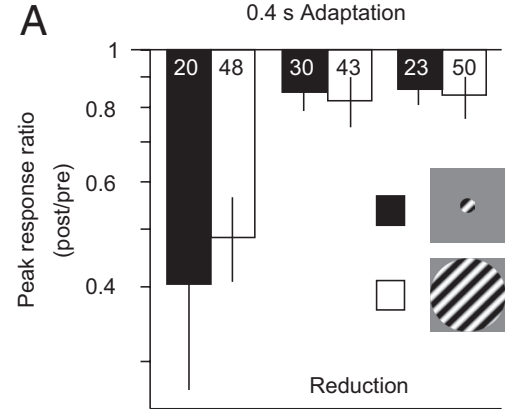

C

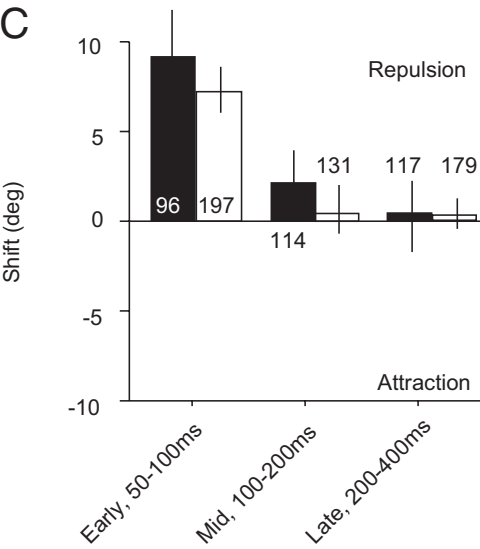

B

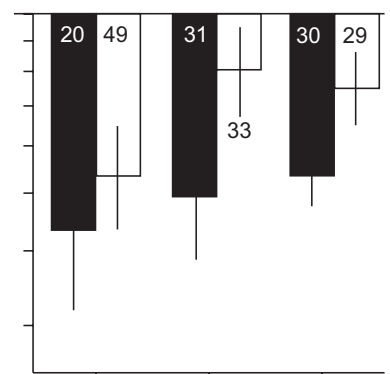

$\mathrm{D}$

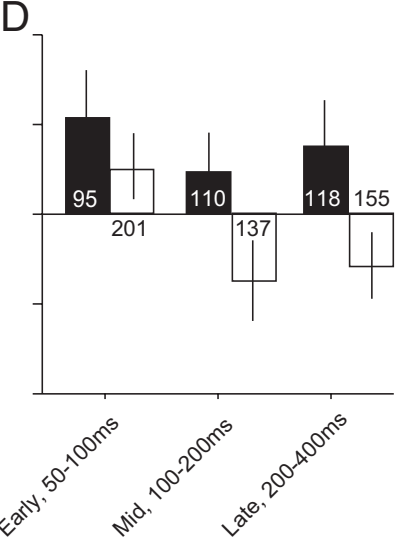

Test response epoch

Figure 5. Effects of brief and prolonged adaptation on different response epochs. $A$, Effect of 0.4 s adaptation on the peak response ratio of preferred adapted neurons, measured 50-100, 100-200, or 200-400 ms after stimulus onset. Data for small gratings are shown in black bars and for large gratings in open bars. Number in/above each bar indicates the number of neurons in each condition. $\boldsymbol{B}$, Effect of 40 s adaptation on the peak response ratio. $\boldsymbol{C}$, Effect of 0.4 s adaptation on the orientation preference of flank-adapted neurons. Because flank-adapted neurons spanned a large range of offsets from the adapter $\left(15-75^{\circ}\right)$, there was a greater number of units for analyzing the shifts in preference than the peak response ratio. $\boldsymbol{D}$, Effect of $40 \mathrm{~s}$ adaptation on the orientation preference of flank-adapted neurons. Error bars indicate $95 \%$ confidence intervals.

mean of $0.40, p=0.0003$ for difference with a ratio of 1 ) or large $(0.48, p<0.0001)$ stimuli, with no significant difference between these conditions $(p=0.34)$. For the middle and late epochs, responses were essentially unaffected by adaptation for both stimulus conditions. After $40 \mathrm{~s}$ adaptation (Fig. 5B), there was again no significant difference in response ratios for small and large stimuli in the early epoch $(0.43$ vs $0.53, p=0.26)$. The ratios were also not significantly different from the corresponding values after brief adaptation $(p>0.4)$. Consistent with previous reports (Lisberger and Movshon, 1999; Priebe et al., 2002), the response ratio in the early epoch was correlated with the transience of the unit (ratio of response in the late epoch compared with early epoch; $r=0.55, p<0.0001$ for brief adaptation; $r=$ $0.31, p=0.02$ for prolonged). Thus, the strongest response reduction in the early epoch was seen for units whose initial response was large compared with that in later epochs.

Notably, during the mid and late epochs, responsivity was strongly reduced after prolonged adaptation for small gratings (0.49 and 0.53 , respectively, $p<0.0001)$ and much less so for large ones (0.81 and $0.75, p<0.05 ; p<0.001$ for comparisons between small and large grating data). Thus, the early epoch is affected similarly by brief and prolonged adaptation, and there is no dependence on stimulus size. An influence of adaptation duration and stimulus size is evident in later epochs, when responses to small but not large gratings are strongly reduced after prolonged adaptation.

We next analyzed the shifts in preference for flank-adapted neurons in each response epoch. After $0.4 \mathrm{~s}$ adaptation (Fig. $5 C$ ), the population level were also statistically significant in this subset of neurons (data not shown).

In summary, adaptation effects appeared similar during the early response epoch, regardless of adapter size or duration. In later epochs, which were only affected by prolonged adaptation, the effects measured with small and large gratings differed. Responses to large gratings were characterized by a weaker loss of responsivity and attractive rather than repulsive shifts in preference.

\section{Testing the dynamics of surround suppression and how it adapts}

The size-dependent effects of prolonged adaptation have been linked to a weakening of surround suppression, recruited by large stimuli that extend beyond the CRF (Wissig and Kohn, 2012). If this is the case, this spatial contextual modulation should be weaker near response onset, to explain the similar effects observed in that epoch for small and large gratings. To test directly how surround suppression influences adaptation-induced changes in orientation tuning, we measured its strength and dynamics, how these are affected by adaptation, and how these relate to changes in tuning in individual units.

We first measured the dynamics of surround suppression before and after adaptation. We compared responses to small gratings $\left(1.3^{\circ}\right.$ diameter $)$ with those evoked by the same stimulus when it was surrounded by an annular grating $\left(2^{\circ}\right.$ inner diameter, $7.4^{\circ}$ outer diameter) of matched orientation. Stimuli were pre- 
sented at five orientations, offset by $0-90^{\circ}$ from the adapter. Neurons that responded to any orientation of the annulus at a rate greater than mean +0.5 SDs of the spontaneous firing rate were discarded; this served to eliminate units for which the annulus encroached on the CRF. Because we wanted to measure the effects of adaptation on surround suppression, we only included units that showed significant preadaptation response suppression $(p<0.05$ for comparison between responses to small gratings presented alone and with an annulus; no correction for multiple comparisons).

Figure $6 A$ shows the population $(n=$ 112) average preadaptation peristimulus time histograms (PSTHs) for the evoked responses of each unit to its preferred orientation of the small stimulus (top trace) and to that same stimulus when it was surrounded by an annulus (bottom trace). Responses were clearly suppressed by the co-presentation of the annulus, but this was only evident $\sim 50 \mathrm{~ms}$ after response onset. The early epoch of the response was similar for the two conditions.

To quantify the strength of surround suppression in each unit, we calculated a suppression index $(\mathrm{SI})$, defined as $\left(r_{\text {small }}\right.$ $-r_{\text {small }}+$ annulus $) / r_{\text {small }}$, where $r_{\text {small }}$ and $r_{\text {small }}+$ annulus are the responses to the small grating and the small grating with annulus, respectively. The SI was calculated using the orientation that evoked the strongest response. Higher SI values indicate stronger surround suppression; negative values indicate that responses were facilitated when the annulus was added. In the early response epoch, 50$100 \mathrm{~ms}$ after stimulus onset, there was no evidence of surround suppression (Fig. $6 B$, bars with thin lines; mean SI, $-0.08, p=0.6$ for difference with 0 ). In all subsequent epochs, suppression was prominent, with a mean value of 0.45 , indicating almost a halving of response strength by the addition of the annulus.

To evaluate how the dynamics of surround suppression were affected by adaptation, we presented an annular adapter for $40 \mathrm{~s}$ and measured responses afterward with our standard top-uptest-protocol. After adaptation, suppression remained negligible in the earliest response epoch (Fig. 6B, bars with thick lines; SI, $-0.02, p=0.9$ ). This would explain why brief adaptation with small and large gratings produced similar effects: brief adaptation affects primarily the early response epoch when surround suppression is minimal. One hundred to $150 \mathrm{~ms}$ after stimulus onset, surround suppression was weaker after adaptation (mean SI of 0.24 compared with 0.53 before adaptation, $p<0.0001$ ). A similar weakening of surround suppression was evident in most subsequent epochs. Measuring surround suppression with responses measured across all epochs revealed a significant reduction in its strength after adaptation (mean SI of 0.42 vs $0.25, p=0.005$ ). Conversely, brief $(0.4 \mathrm{~s})$ adaptation with an annulus had a mini-
B

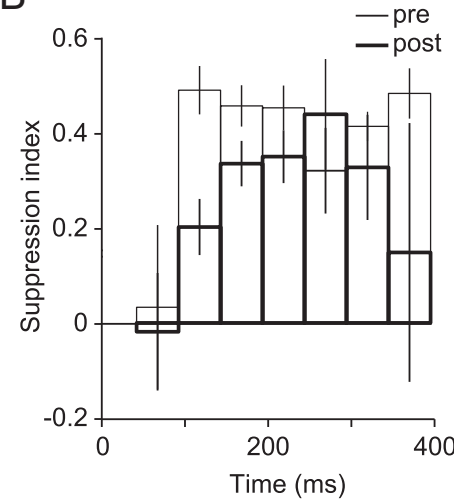

$\mathrm{D}$

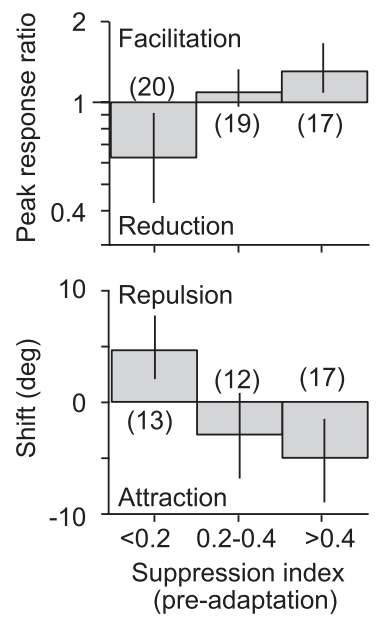

Figure 6. Surround suppression: dynamics and effects of adaptation. $\boldsymbol{A}$, Population average PSTHs for responses to a preferred, smaling and to the same stimulus when surrounded by an annulus of matched orientation. We normalized the PSTH for each unit by its peak response and then averaged across units. Error bars indicate SEM. B, SI before (thin) and after (thick) 40 s adaptation 政 ratio in preferred-adapted neurons (top) and shifts in preferred orientation in flank-adapted neurons (bottom), after 40 s adaptation with an annular grating. Data are plotted as a function of the preadaptation SI. Error bars indicate $95 \%$ confidence levels.

mal effect on the SI $(0.45$ vs $0.42, p=0.02, n=136$; data not shown).

The adaptation-induced changes in surround suppression in Figure $6 B$ were based on responses of each unit to its preferred stimulus, regardless of its orientation offset to the annular adapter. To test the specificity of these effects, we plot the SI difference (post - pre) as a function of the preferred orientation of the unit relative to the adapter. The most profound weakening of surround suppression occurred for neurons whose preferred orientation matched the annular adapter: the mean SI difference was -0.34 (from 0.44 to $0.10, p=0.04$ ), representing an almost complete elimination of surround suppression in this subset of neurons. The effect was weaker for neurons whose preferred orientation was offset from the adapter and negligible for neurons with offsets $>45^{\circ}$. The weakening of surround suppression by annular adaptation is thus greatest when the orientation of the preferred stimulus of a neuron is matched to the adapter.

If weakened surround suppression contributes to changes in tuning after prolonged adaptation with large gratings, we would expect the changes in tuning in each neuron to depend on how strongly it was surround suppressed. We therefore measured the dependence of the response ratio and shifts in preference on the 
A $0.4 \mathrm{~s}$ adaptation Delay $=0 \mathrm{~s}$

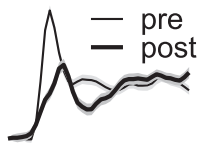

Delay $=0.02 \mathrm{~s}$

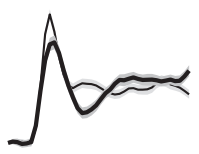

Delay $=0.1 \mathrm{~s}$

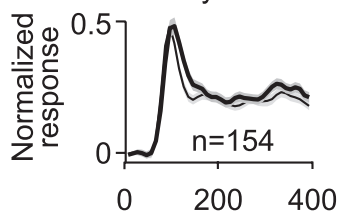

B

$4 \mathrm{~s}$ adaptation

Delay $=0 \mathrm{~s}$

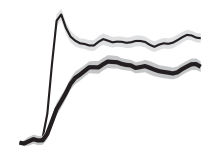

Delay $=1 \mathrm{~s}$

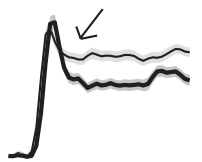

Delay $=4 \mathrm{~s}$

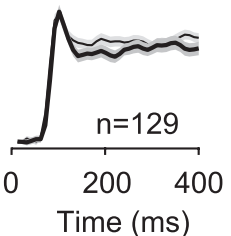

C

$40 \mathrm{~s}$ adaptation

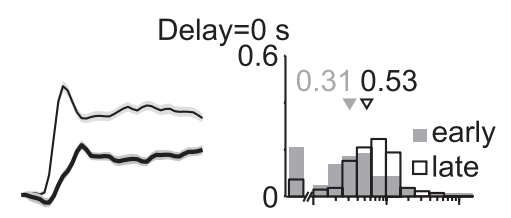

Delay $=40 \mathrm{~s}$

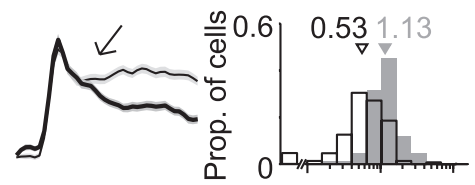

Delay $=120 \mathrm{~s}$

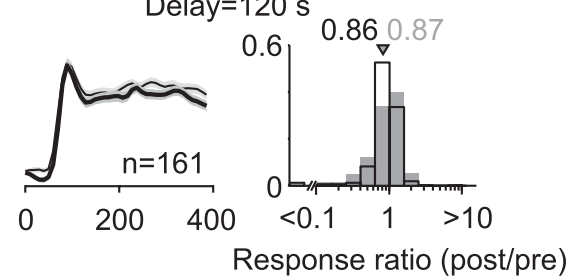

Figure 7. Time course of recovery from adaptation. A, Population average PSTHs before (thin black lines with light gray shading for SEM) and after (thick black lines surrounded by darker shading) $0.4 \mathrm{~s}$ adaptation. Postadaptation responses were measured after the indicated delay (left) and are superimposed on the preadaptation responses for ease of comparison. Abscissa indicates time relative to test stimulus onset. $\boldsymbol{B}$, Recovery from $4 \mathrm{~s}$ adaptation, following the convention of $\boldsymbol{A}$. Arrow highlights the rapid recovery of the initial response epoch, relative to later response epochs. $\boldsymbol{C}$, Recovery from $40 \mathrm{~s}$ adaptation. Arrow highlights the rapid recovery of the initial response epoch, relative to later response epochs. Right, Response ratios (postadaptation/preadaptation) measured immediately after 40 s adapter offset (top), 40 s later (middle), or 120 s later (bottom). Ratios measured 50 - 100 ms after stimulus onset are shown in gray shading; those measured 200 - $400 \mathrm{~ms}$ after stimulus onset are shown in open bars. PSTH error bars indicate SEM.

preadaptation SI. Tuning was measured with small gratings surrounded by an annulus, before and after a 40 s presentation of an annular adapter. All units, regardless of preadaptation SI, were included in this analysis so that the effects on tuning in the full population could be observed. However, we only considered units whose tuning was well fit by a von Mises function over the limited $\left(90^{\circ}\right)$ range of orientations sampled (for criteria, see Materials and Methods).

Changes in responsivity in preferred-adapted neurons were related to the strength of surround suppression (Fig. $6 D ; n=56$, $F=6.9, p=0.002$, ANOVA). Units with strong surround suppression $(\mathrm{SI}>0.4)$ facilitated after adaptation (mean response ratio of $1.31, p=0.02$ ), whereas those with weak or no suppression $(\mathrm{SI}<0.2)$ showed a loss of responsivity $(0.62, p=0.03)$. Similarly, shifts in preference in flank-adapted units were related to the preadaptation SI $(n=42, F=7.2, p=0.002)$. The most strongly surround-suppressed units showed the largest attractive shifts in preference (mean of $-5.0^{\circ}, p=0.02$ ), and those with no surround suppression were repelled away from the adapter $\left(4.6^{\circ}\right.$, $p=0.01)$.

Interestingly, in units with little surround suppression (SI < 0.2 ), the annular adapter caused a loss of responsivity and repulsive shifts. Although we only analyzed responses from units for which the annulus caused no measurable change in firing rate, the annulus presumably could still impinge on an excitatory region of the CRF that was insufficient by itself to drive the units to threshold. A stimulus-specific reduction of this input after annular adaptation would reduce responsivity and repel tuning, as we observed.

In summary, surround suppression was absent in the initial response epoch, explaining why brief adaptation effects are size independent. Annular adaptation reduced the strength of surround suppression evident in later response epochs, particularly for neurons for which the annulus orientation was that preferred within the CRF. The strength of surround suppression was related to the manner in which adaptation altered orientation tuning, on a unit-by-unit basis.

\section{Testing how adaptation duration affects response recovery}

Duration scaling suggests that more prolonged adaptation simply strengthens effects seen after brief adaptation. Our results are clearly inconsistent with this description. Duration scaling also suggests that more prolonged adaptation results in more persistent effects. To test this, we measured how quickly neuronal responses recover after adapter offset. We were particularly interested in comparing effects in different response epochs, because these show different sensitivity to adaptation. Does the early epoch, which is affected most readily by adaptation, also reappear most quickly? Or does the late response epoch, most resistant to adaptation and only affected by the most prolonged adapters, recover first?

We measured responses to a large test grating identical to the adapter, at a range of temporal delays after adapter offset (see Materials and Methods). Figure $7 A-C$ shows the population average PSTHs of evoked responses, measured before adaptation (thin black lines) and at the indicated time point after adapter offset (thick lines). After $0.4 \mathrm{~s}$ adaptation (Fig. $7 A ; n=154$ ), primarily the early response epoch was reduced in amplitude, but this recovered within $0.1 \mathrm{~s}$. After $4 \mathrm{~s}$ adaptation (Fig. $7 B ; n=129$ ), the early epoch was reduced in amplitude, and later epochs were affected as well. Responses had primarily recovered within $4 \mathrm{~s}$. After $40 \mathrm{~s}$ adaptation (Fig. $7 C ; n=161$ ), responsivity in all epochs was reduced, and full recovery occurred after $120 \mathrm{~s}$.

During recovery, the adaptation effects in the early response epoch dissipated more quickly than those in later epochs. This was particularly apparent $40 \mathrm{~s}$ after offset of the $40 \mathrm{~s}$ adapter (Fig. 
$7 C$, arrow) but was also evident $1 \mathrm{~s}$ after the offset of the $4 \mathrm{~s}$ adapter (Fig. $7 B$, arrow). We compared this directly by measuring, for each unit, the response ratios for the early (50-100 ms) and late (200$400 \mathrm{~ms}$ ) epochs at different time points after the offset of the $40 \mathrm{~s}$ adapter (Fig. 7C; right column). Immediately after adaptation, the responsivity was strongly reduced in both epochs (ratios of 0.31 and $0.53, p=0.003$ for difference). At $40 \mathrm{~s}$ after adapter offset, responses in the early epoch had recovered (ratio of 1.13), whereas those in the late epoch had not (ratio of $0.53, p<0.0001$ for difference). After $120 \mathrm{~s}$ of recovery, responsivity in both epochs had largely recovered.

To quantify the rate of recovery, we fit a rising exponential to the early and late response epochs of each unit (see Materials and Methods). Recovery of an example cell from $40 \mathrm{~s}$ adaptation is shown in Figure $8 A$ for both the early (gray) and late (black) response epochs, which were fit separately. The fits accounted for the majority of the variance in both epochs (85 and $97 \%$, respectively), but the time constant was substantially shorter for the early (12.8s) compared with the late (37.2 s) epoch.

To quantify effects in the population, we included units whose recovery in both the early and late epochs was well fit ( $>50 \%$ variance accounted for), so that these could be compared directly. On average, the fits accounted for $75 \%$ of the response variance. Average values of the time constant of the exponential recovery are shown in Figure 8. For $0.4 \mathrm{~s}$ adaptation (Fig. $8 B$ ), the mean time constant was $130 \mathrm{~ms}$ for the early response epoch. Because brief adaptation did not affect the later epoch, we could not measure the recovery rate. After $4 \mathrm{~s}$ adaptation (Fig. 8C), the recovery of the early epoch slowed (gray histogram, mean of $750 \mathrm{~ms}$ ), but it was more rapid than the recovery of late epoch (open bars, $1.70 \mathrm{~s}$; paired $t$ test, $p<0.0001$ for difference). An additional slowing of recovery was observed after $40 \mathrm{~s}$ adaptation (Fig. $8 D$ ), but the early epoch recovered more quickly than the later epoch (19.6 s compared with $57.1 \mathrm{~s}, p<0.0001$ ). When plotted on log-log axes (Fig. $8 E$ ), the time constant of recovery displayed a nearly linear dependence on adaptation duration. Thus, recovery time was consistent with a power-law dependence on adaptation duration (Drew and Abbott, 2006; Wark et al., 2007), although given our limited sampling, we cannot draw a definitive conclusion.

In summary, although prolonged adaptation does not strengthen effects in the early response epoch, it does make those effects more persistent, consistent with duration scaling. However, the early epoch recovers more quickly than the late epoch, for all adaptation durations.

\section{Summary of effects}

Figure 9 provides a schematic summary of the effects we observed. In the early response epoch (Fig. 9A), small gratings cause a similar loss of responsivity and shifts in preference for all adaptation durations (top). Effects are independent of adaptation duration. In this epoch, surround suppression is essentially absent
B $0.4 \mathrm{~s}$ adaptation

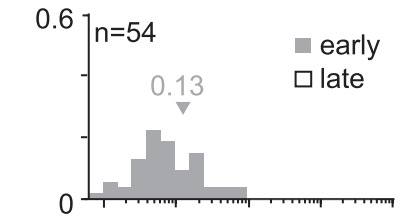

C
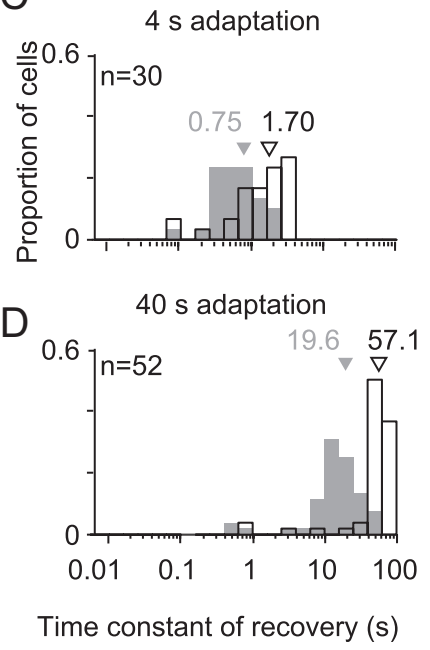

Time constant of recovery (s)

Adaptation duration (s)

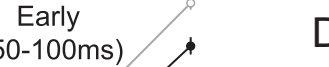

Late

$(200-400 \mathrm{~ms})$

40

Figure 8. Time constant of recovery. $\boldsymbol{A}$, Recovery of an example unit after 40 s adaptation for the early (gray) and late (black) Data points show observed response as a function of time elapsed from offset of the adapter. Solid lines show fits of the constant of recovery after $0.4 s(B), 4 s(C)$, and $40 s(D)$ adaptation. The time constant measured for the early response (50-100 ms after stimulus onset) are shown in gray shading and for the late response ( $200-400 \mathrm{~ms}$ ) as open for the early (gray line) and late (black) epochs, as a function of adaptation duration.

so annular adaptation - which targets the surround - has no effect (middle). For this reason, tuning measured with large gratings (bottom) is altered by adaptation in the same manner as for small gratings.

In later epochs (Fig. 9B), effects on tuning measured with small gratings are consistent with duration scaling: response reduction and repulsive shifts increase in strength with more prolonged adaptation (top row). For large gratings (bottom row), effects reflect the combined influence of altered responses within the CRF and weakened surround suppression (middle row), yielding weak response reduction and attractive shifts after prolonged adaptation. The loss of peak responsivity is thus independent of adaptation duration, and shifts in preference switch from being repulsive to attractive for more prolonged adaptation.

\section{Discussion}

We measured the effects on V1 orientation tuning of adaptation varying from $0.4 \mathrm{~s}$, approximately the duration of a single visual fixation, to $40 \mathrm{~s}$, typical of the duration used in psychophysical and imaging studies - a 100-fold range. Brief and prolonged adaptation could have qualitatively different effects, depending on stimulus size and the response epoch analyzed. Our results show that duration scaling is a poor description of how neuronal responses are influenced by adaptation duration. Furthermore, they suggest distinct strategies for adjusting to moment-tomoment fluctuations in the visual environment and to more persistent input.

Duration scaling indicates that adaptation duration influences the magnitude and persistence of effects. Our results provide two clear violations of this description. First, prolonged and brief adaptation with large gratings caused opposite shifts in pref- 
A

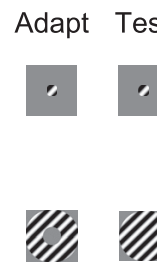

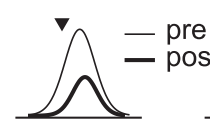
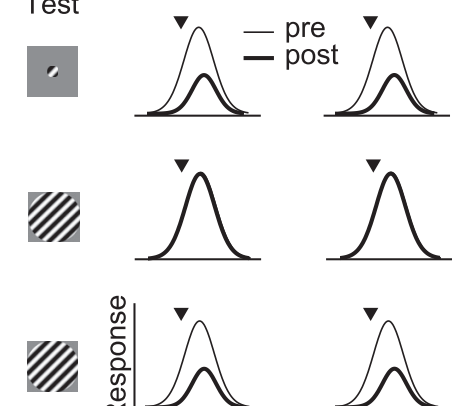

Early response epoch
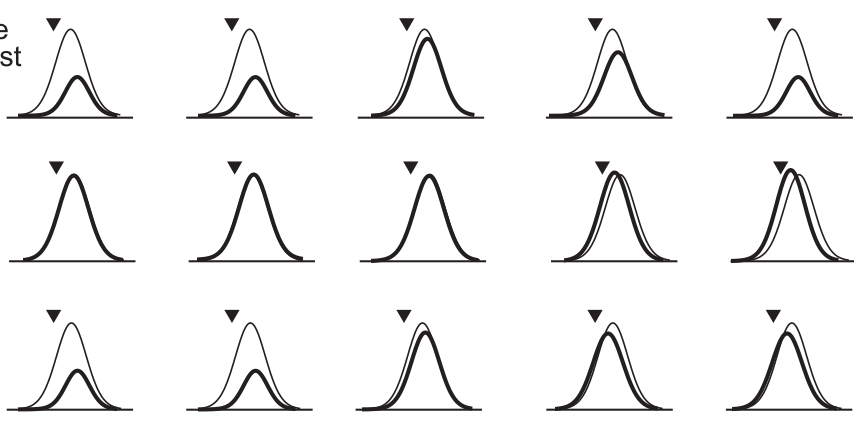

$\overleftarrow{\text { Brief }}$ Adaptation duration Prolonged Brie Prolonged

Figure 9. Summary of how adaptation alters orientation tuning, for different stimuli and adaptation durations. $\boldsymbol{A}$, Effects in the early response epoch. $\boldsymbol{B}$, Effects in later epochs. Thin lines indicate preadaptation responses, and thick lines indicate postadaptation.

erence and indistinguishable changes in responsivity (Figs. 2, 3). Second, adaptation duration had no consequence on the magnitude of effects in the early response epoch (Fig. 5), independent of stimulus size. To our knowledge, only a single previous study evaluated how adaptation duration affects changes in neuronal tuning. Dragoi et al. (2000) explored a complementary range of timescales and reported stronger repulsive shifts in V1 orientation tuning with $10 \mathrm{~min}$ than $10 \mathrm{~s}$ adaptation. They also found that flank adaptation lasting $2 \mathrm{~min}$ or more caused responses on the opposite flank to increase, consistent with our results that more prolonged adaptation may recruit effects not seen after briefer exposures (see also Ghisovan et al., 2009).

Our finding that shifts in preference reverse sign with more prolonged adaptation appears to involve a weakening of the surround suppression recruited by large gratings. We previously reported attractive shifts in V1 tuning (Wissig and Kohn, 2012), an effect first observed in area MT (Kohn and Movshon, 2004; Krekelberg et al., 2006). We suggested that this involves a stimulus-specific weakening of surround suppression. Here we provide direct support for this mechanism: units with strong surround suppression showed a facilitation of responsivity and attractive shifts in preference after adaptation with an annular grating, whereas those with little surround suppression showed a loss of responsivity and repulsive shifts in preference.

Although prolonging adaptation caused a reversal of shifts in preference, it did not cause a greater loss of responsivity in preferred-adapted neurons. This could be explained by a difference in the stimulus specificity of effects in the surround and CRF. Extending adaptation duration leads to progressively stronger effects within the CRF (Figs. 2, 3). For preferred-adapted units, a concomitant progressive weakening of the surround would cause a disinhibition that could negate the loss of responsivity for stimuli matched to the adapter. However, if the stimulus specificity of effects in the CRF and surround differ, shifts in tuning can change in magnitude or sign with adaptation duration, as we observed.

The weakening of surround suppression we observe after prolonged adaptation is consistent with several recent studies showing normalization signals from within (Dhruv et al., 2011) and outside (Tailby et al., 2008; Camp et al., 2009; Wissig and Kohn, 2012) the CRF can be weakened by adaptation. These signals may adapt at different rates and also differently from the excitatory drive provided within the CRF. As a result, the influence of adaptation duration will likely depend on which excitatory and suppressive signals are recruited by a particular stimulus. In our measurements, the influence of stimulus size was only apparent after prolonged adaptation. Brief adaptation affected primarily the early response epoch, when surround suppression was minimal.

The second violation of duration scaling was evident in the early response epoch, which was affected similarly by brief and prolonged adaptation for both small and large gratings. The early epoch has high contrast and response gain (Müller et al., 2001), is relatively unaffected by spatial contextual modulation (Bair et al., 2003; Xing et al., 2005; Sugihara et al., 2011; Fig. 6), and may underlie rapid perceptual decisions (Thorpe et al., 1996; Shriki et al., 2012). The quick induction and recovery of effects in this epoch could serve to adjust sensitivity from fixation to fixation. When successive fixations fall on similar features, the initial response triggered by the second fixation will be strongly reduced, because of both a loss of responsivity in preferred-adapted neurons and a shift in preference for flank-adapted ones. This would serve to suppress the representation of redundant visual input.

In contrast, responses in later epochs are only affected by persistent visual input, perhaps contributing to a more stable neuronal representation (Liu et al., 2009). Adaptation effects in later epochs are strongly modulated by spatial context. Whether the neuronal representation to a feature is reduced, maintained, or enhanced (by shifting tuning toward the persistent feature) depends critically on the spatial context in which that feature is embedded. The full functional meaning of the strong interaction between temporal (adaptation) and spatial contextual modulation remains to be established. Their entwinement may suggest a common purpose, such as determining stimulus salience, a function traditionally attributed to spatial contextual influence alone (Li, 1999; Itti and Koch, 2000).

\section{Relationship to perceptual findings}

In perceptual studies, duration scaling often takes the form of a linear or logarithmic increase in effect strength with the logarithm of adaptation duration (Greenlee and Magnussen, 1987; Greenlee et al., 1991; Leopold et al., 2005). We observed such a relationship only for responses to small gratings measured over several hundred milliseconds (Figs. $2 B, 3 B$ ). Do the violations of 
duration scaling we observe thus suggest an inconsistency between V1 neuronal effects and perceptual experiments? Not necessarily. The best-studied examples of duration scaling for grating adaptation are the elevation of contrast detection thresholds (Greenlee et al., 1991) and the TAE (Greenlee and Magnussen 1987). Detection thresholds are measured, by definition, with low-contrast stimuli that recruit minimal surround influence (Sceniak et al., 1999; Cavanaugh et al., 2002). If these stimuli are presented for at least a few hundred milliseconds, the responses they evoke would be expected to become weaker after more prolonged adaptation. The strength of the TAE is determined by both changes in neuronal responsivity and tuning (Kohn and Movshon, 2004; Jin et al., 2005), with attractive shifts in preference leading to stronger effects. Our results thus predict the TAE measured with both small and large gratings should increase with adaptation duration, as reported in perceptual studies.

Our observations do provide a direct correlate for a number of previous perceptual findings. First, several studies have found that brief and prolonged adaptation can have equally strong effects, when a test stimulus is sufficiently brief (Harris and Calvert, 1989; Foley and Boynton, 1993; Pavan et al., 2012). This is consistent with our findings, if one assumes that brief stimuli only evoked the initial response measured in our experiments. Second, perceptual recovery has a power function dependence on adaptation duration and is essentially independent of the strength of an aftereffect (Greenlee et al., 1991). Similarly, we found that recovery time depends on adaptation duration but not strength of effect (i.e., for the early response epoch), following a power function relationship. Third, repeated presentations of brief adapters induce stronger perceptual effects than continuous adaptation of the same total duration (Magnussen and Greenlee, 1986). This could be explained by the rapid recovery of the initial response epoch, allowing each brief adapter to provide stronger transient neuronal responses and thus inducing stronger effects in later epochs.

\section{Timescales of adaptation}

More generally, the violations of duration scaling and the dynamics of recovery suggest that adaptation effects reflect a multitimescale process. This is consistent with several recent perceptual and neurophysiological studies. For instance, Vul et al. (2008) showed that the McCollough effect, a well-known aftereffect induced by the contingent presentation of two stimulus features, consists of a fast saturating component and a slow nonsaturating component, which sum linearly. Bao and Engel (2012) showed that the TAE induced by adaptation of $>1 \mathrm{~h}$ can be transiently "erased" by the brief presentation of a counteradapter. As the effects of the brief counter-adapter dissipated, the original aftereffect reappeared. This suggests the storage of aftereffects with multiple timescales within a neuron or network.

Neurophysiological evidence for multiple timescales of adaptation includes the existence of rapid $(<0.1 \mathrm{~s})$ and slower $(\sim 10 \mathrm{~s})$ contrast adaptation effects in the retina (Baccus and Meister, 2002). Wark et al. (2009) also showed that retinal adaptation effects are induced more rapidly when the statistics of a stimulus ensemble change frequently (i.e., brief exposure to a particular ensemble) and suggested that this reflects an inference about the current state of the environment: longer exposures to an ensemble result in a firmer belief in the current state and thus a slower adjustment of the network when the input changes. Our results show that cortical adaptation effects display similar, multitimescale behavior and thus may reflect a sophisticated internal model of the environment.
The multi-timescale behavior we report could be the result of individual cellular mechanisms that operate over multiple timescales: recovery from sodium channel inactivation has a power law dependence on stimulus duration (Toib et al., 1998; Gilboa et al., 2005), and synaptic depression involves both rapid (hundreds of milliseconds) and slow (tens of seconds) components (Varela et al., 1997; Chance et al., 1998). However, it is more likely that multi-timescale behavior is mediated by diverse mechanisms that are recruited and dissipate at different rates. For instance, rapid synaptic depression may contribute to the rapidly induced effects observed in the early epoch. In contrast, postsynaptic hyperpolarization requires the accumulation of intracellular ions (Carandini and Ferster, 1997; Sanchez-Vives et al., 2000a,b) and may thus only be recruited by more persistent input. However, this simple explanation would suggest that effects in the early epoch would grow with adaptation duration, because the contribution of hyperpolarization and reduced synaptic input would sum. This is inconsistent with our results and indicates a more complex interplay of adaptation mechanisms, which remain poorly understood for adaptation in general (for discussion, see Kohn, 2007).

In summary, the nervous system adjusts to the environment over a very wide range of timescales. This plasticity has been parceled into distinct categories of study-adaptation, perceptual learning, reorganization after central or peripheral injury (e.g., retinal lesion studies), development, and so on-which emphasize distinct phenomenology. For instance, adaptation is often found to reduce responsivity and the representation of recently encountered stimuli, but frequent exposure to a stimulus during development or adulthood can lead to its overrepresentation in cortex (Fahle and Poggio, 2002; Lu et al., 2009; Webster, 2011). Thus, it is clear that cortex has different strategies for adjusting to the environment on the gross timescales that separate these categories of sensory-driven plasticity. Our results suggest that, within a range of durations falling under the term adaptation, different effects and strategies also exist.

\section{References}

Angelucci A, Bressloff PC (2006) Contribution of feedforward, lateral and feedback connections to the classical receptive field center and extraclassical receptive field surround of primate V1 neurons. Prog Brain Res 154:93-120. CrossRef Medline

Baccus SA, Meister M (2002) Fast and slow contrast adaptation in retinal circuitry. Neuron 36:909-919. CrossRef Medline

Bair W, Cavanaugh JR, Movshon JA (2003) Time course and time-distance relationships for surround suppression in macaque V1 neurons. J Neurosci 23:7690-7701. Medline

Bao M, Engel SA (2012) Distinct mechanism for long-term contrast adaptation. Proc Natl Acad Sci U S A 109:5898-5903. CrossRef Medline

Bonds AB (1991) Temporal dynamics of contrast gain in single cells of the cat striate cortex. Vis Neurosci 6:239-255. CrossRef Medline

Camp AJ, Tailby C, Solomon SG (2009) Adaptable mechanisms that regulate the contrast response of neurons in the primate lateral geniculate nucleus. J Neurosci 29:5009-5021. CrossRef Medline

Carandini M, Ferster D (1997) A tonic hyperpolarization underlying contrast adaptation in cat visual cortex. Science 276:949-952. CrossRef Medline

Cavanaugh JR, Bair W, Movshon JA (2002) Nature and interaction of signals from the receptive field center and surround in macaque V1 neurons. J Neurophysiol 88:2530-2546. CrossRef Medline

Chance FS, Nelson SB, Abbott LF (1998) Synaptic depression and the temporal response characteristics of V1 cells. J Neurosci 18:4785-4799. Medline

Clifford CW, Webster MA, Stanley GB, Stocker AA, Kohn A, Sharpee TO, Schwartz O (2007) Visual adaptation: neural, psychological and computational aspects. Vision Res 47:3125-3131. CrossRef Medline

Crowder NA, Hietanen MA, Price NS, Clifford CW, Ibbotson MR (2008) 
Dynamic contrast change produces rapid gain control in visual cortex. J Physiol 586:4107-4119. CrossRef Medline

Dhruv NT, Tailby C, Sokol SH, Lennie P (2011) Multiple adaptable mechanisms early in the primate visual pathway. J Neurosci 31:15016-15025. CrossRef Medline

Dragoi V, Sharma J, Sur M (2000) Adaptation-induced plasticity of orientation tuning in adult visual cortex. Neuron 28:287-298. CrossRef Medline

Dragoi V, Rivadulla C, Sur M (2001) Foci of orientation plasticity in visual cortex. Nature 411:80-86. CrossRef Medline

Dragoi V, Sharma J, Miller EK, Sur M (2002) Dynamics of neuronal sensitivity in visual cortex and local feature discrimination. Nat Neurosci 5:883-891. CrossRef Medline

Drew PJ, Abbott LF (2006) Models and properties of power-law adaptation in neural systems. J Neurophysiol 96:826-833. CrossRef Medline

El-Shamayleh Y, Movshon JA (2011) Neuronal responses to texturedefined form in macaque visual area V2. J Neurosci 31:8543-8555. CrossRef Medline

Fahle M, Poggio TA (2002) Perceptual learning. Cambridge, MA: Massachusetts Institute of Technology.

Felleman DJ, Van Essen DC (1991) Distributed hierarchical processing in the primate cerebral cortex. Cereb Cortex 1:1-47. CrossRef Medline

Felsen G, Shen YS, Yao H, Spor G, Li C, Dan Y (2002) Dynamic modification of cortical orientation tuning mediated by recurrent connections. Neuron 36:945-954. CrossRef Medline

Foley JM, Boynton GM (1993) Forward pattern masking and adaptation: effects of duration, interstimulus interval, contrast, and spatial and temporal frequency. Vision Res 33:959-980. CrossRef Medline

Foster KH, Gaska JP, Nagler M, Pollen DA (1985) Spatial and temporal frequency selectivity of neurones in visual cortical areas V1 and V2 of the macaque monkey. J Physiol 365:331-363. Medline

Ghisovan N, Nemri A, Shumikhina S, Molotchnikoff S (2009) Long adaptation reveals mostly attractive shifts of orientation tuning in cat primary visual cortex. Neuroscience 164:1274-1283. CrossRef Medline

Gilboa G, Chen R, Brenner N (2005) History-dependent multiple-timescale dynamics in a single-neuron model. J Neurosci 25:6479-6489. CrossRef Medline

Greenlee MW, Magnussen S (1987) Saturation of the tilt aftereffect. Vision Res 27:1041-1043. CrossRef Medline

Greenlee MW, Georgeson MA, Magnussen S, Harris JP (1991) The time course of adaptation to spatial contrast. Vision Res 31:223-236. CrossRef Medline

Harris JP, Calvert JE (1989) Contrast, spatial frequency and test duration effects on the tilt aftereffect: implications for underlying mechanisms. Vision Res 29:129-135. CrossRef Medline

Hensch TK (2005) Critical period plasticity in local cortical circuits. Nat Rev Neurosci 6:877-888. CrossRef Medline

Itti L, Koch C (2000) A saliency-based search mechanism for overt and covert shifts of visual attention. Vision Res 40:1489-1506. CrossRef Medline

Jin DZ, Dragoi V, Sur M, Seung HS (2005) Tilt aftereffect and adaptationinduced changes in orientation tuning in visual cortex. J Neurophysiol 94:4038-4050. CrossRef Medline

Kohn A (2007) Visual adaptation: physiology, mechanisms, and functional benefits. J Neurophysiol 97:3155-3164. CrossRef Medline

Kohn A, Movshon JA (2004) Adaptation changes the direction tuning of macaque MT neurons. Nat Neurosci 7:764-772. CrossRef Medline

Krekelberg B, van Wezel RJ, Albright TD (2006) Adaptation in macaque MT reduces perceived speed and improves speed discrimination. J Neurophysiol 95:255-270. CrossRef Medline

Leopold DA, Rhodes G, Müller KM, Jeffery L (2005) The dynamics of visual adaptation to faces. Proc Biol Sci 272:897-904. CrossRef Medline

Li Z (1999) Contextual influences in V1 as basis for pop out and asymmetry in visual search. Proc Natl Acad Sci U S A 96:10530-10535. CrossRef Medline

Lisberger SG, Movshon JA (1999) Visual motion analysis for pursuit eye movements in area MT of macaque monkeys. J Neurosci 19:2224-2246. Medline

Liu Y, Murray SO, Jagadeesh B (2009) Time course and stimulus dependence of repetition-induced response suppression in inferotemporal cortex. J Neurophysiol 101:418-436. CrossRef Medline

Lu ZL, Yu C, Watanabe T, Sagi D, Levi D (2009) Perceptual learning: func- tions, mechanisms, and applications. Vision Res 49:2531-2534. CrossRef Medline

Magnussen S, Greenlee MW (1986) Contrast threshold elevation following continuous and interrupted adaptation. Vision Res 26:673-675. CrossRef Medline

Magnussen S, Johnsen T (1986) Temporal aspects of spatial adaptation. A study of the tilt aftereffect. Vision Res 26:661-672. CrossRef Medline

Müller JR, Metha AB, Krauskopf J, Lennie P (1999) Rapid adaptation in visual cortex to the structure of images. Science 285:1405-1408. CrossRef Medline

Müller JR, Metha AB, Krauskopf J, Lennie P (2001) Information conveyed by onset transients in responses of striate cortical neurons. J Neurosci 21:6978-6990. Medline

Ohzawa I, Sclar G, Freeman RD (1985) Contrast gain control in the cat's visual system. J Neurophysiol 54:651-667. Medline

Pavan A, Marotti RB, Campana G (2012) The temporal course of recovery from brief (sub-second) adaptations to spatial contrast. Vision Res 62C: 116-124. CrossRef Medline

Priebe NJ, Lisberger SG (2002) Constraints on the source of short-term motion adaptation in macaque area MT. II. Tuning of neural circuit mechanisms. J Neurophysiol 88:370-382. Medline

Priebe NJ, Churchland MM, Lisberger SG (2002) Constraints on the source of short-term motion adaptation in macaque area MT. I. The role of input and intrinsic mechanisms. J Neurophysiol 88:354-369. Medline

Sanchez-Vives MV, Nowak LG, McCormick DA (2000a) Membrane mechanisms underlying contrast adaptation in cat area 17 in vivo. J Neurosci 20:4267-4285. Medline

Sanchez-Vives MV, Nowak LG, McCormick DA (2000b) Cellular mechanisms of long-lasting adaptation in visual cortical neurons in vitro. J Neurosci 20:4286-4299. Medline

Sceniak MP, Ringach DL, Hawken MJ, Shapley R (1999) Contrast's effect on spatial summation by macaque V1 neurons. Nat Neurosci 2:733-739. CrossRef Medline

Sharpee TO, Sugihara H, Kurgansky AV, Rebrik SP, Stryker MP, Miller KD (2006) Adaptive filtering enhances information transmission in visual cortex. Nature 439:936-942. CrossRef Medline

Shriki O, Kohn A, Shamir M (2012) Fast coding of orientation in primary visual cortex. PLoS Comput Biol 8:e1002536. CrossRef Medline

Stocker AA, Simoncelli EP (2006) Noise characteristics and prior expectations in human visual speed perception. Nat Neurosci 9:578-585. CrossRef Medline

Sugihara T, Qiu FT, von der Heydt R (2011) The speed of context integration in the visual cortex. J Neurophysiol 106:374-385. CrossRef Medline

Suzuki S (2001) Attention-dependent brief adaptation to contour orientation: a high-level aftereffect for convexity? Vision Res 41:3883-3902. CrossRef Medline

Suzuki S (2005) High-level pattern coding revealed by brief shape aftereffects. Fitting the mind to the world: adaptation and after-effects in high level vision (Clifford CW, Rhodes G, eds), pp 135-172. New York: Oxford UP.

Tailby C, Solomon SG, Lennie P (2008) Functional asymmetries in visual pathways carrying S-cone signals in macaque. J Neurosci 28:4078-4087. CrossRef Medline

Thorpe S, Fize D, Marlot C (1996) Speed of processing in the human visual system. Nature 381:520-522. CrossRef Medline

Toib A, Lyakhov V, Marom S (1998) Interaction between duration of activity and time course of recovery from slow inactivation in mammalian brain $\mathrm{Na}^{+}$channels. J Neurosci 18:1893-1903. Medline

Varela JA, Sen K, Gibson J, Fost J, Abbott LF, Nelson SB (1997) A quantitative description of short-term plasticity at excitatory synapses in layer $2 / 3$ of rat primary visual cortex. J Neurosci 17:7926-7940. Medline

Vul E, Krizay E, MacLeod DI (2008) The McCollough effect reflects permanent and transient adaptation in early visual cortex. J Vis 8(12):4 1-12. CrossRef

Wark B, Lundstrom BN, Fairhall A (2007) Sensory adaptation. Curr Opin Neurobiol 17:423-429. CrossRef Medline

Wark B, Fairhall A, Rieke F (2009) Timescales of inference in visual adaptation. Neuron 61:750-761. CrossRef Medline

Webster MA (2011) Adaptation and visual coding. J Vis 11(5):3 1-23. CrossRef

Wissig SC, Kohn A (2012) The influence of surround suppression on adap- 
tation effects in primary visual cortex. J Neurophysiol 107:3370-3384. CrossRef Medline

Xing D, Shapley RM, Hawken MJ, Ringach DL (2005) Effect of stimulus size on the dynamics of orientation selectivity in macaque V1. J Neurophysiol 94:799-812. CrossRef Medline 